This is the author's final, peer-reviewed manuscript as accepted for publication. The publisher-formatted version may be available through the publisher's web site or your institution's library.

\title{
Neighborhood-level socioeconomic and urban land use risk factors of canine leptospirosis: 94 cases (2002-2009)
}

R. K. Raghavan, K. M. Brenner, J. J. Higgins, J. M. Shawn Hutchinson, K. R. Harkin

\section{How to cite this manuscript}

If you make reference to this version of the manuscript, use the following information:

Raghavan, R. K., Brenner, K. M., Higgins, J. J., Hutchinson, J. M. S., \& Harkin, K. R. (2012). Neighborhood-level socioeconomic and urban land use risk factors of canine leptospirosis: 94 cases (2002-2009). Retrieved from http://krex.ksu.edu

\section{Published Version Information}

Citation: Raghavan, R. K., Brenner, K. M., Higgins, J. J., Hutchinson, J. M. S., \& Harkin, K. R. (2012). Neighborhood-level socioeconomic and urban land use risk factors of canine leptospirosis: 94 cases (2002-2009). Preventive Veterinary Medicine, 106(34), 324-331.

Copyright: ( 2012 Elsevier B.V.

Digital Object Identifier (DOI): doi:10.1016/j.prevetmed.2012.04.003

Publisher's Link: http://www.sciencedirect.com/science/article/pii/S0167587712001341

This item was retrieved from the K-State Research Exchange (K-REx), the institutional repository of Kansas State University. K-REx is available at http://krex.ksu.edu 
1 Title: Neighborhood-level socioeconomic and urban land use risk factors of canine leptospirosis.

294 cases (2002-2009).

3

4 R.K. Raghavan ${ }^{\mathrm{a},{ }^{*},}$, K.M. Brenner ${ }^{\mathrm{b}}$, J.J. Higgins ${ }^{\mathrm{c}}$, J.M. Shawn Hutchinson ${ }^{\mathrm{d}}$, K.R. Harkin ${ }^{\mathrm{b}}$. 5

$6{ }^{a}$ Kansas State Veterinary Diagnostic Laboratory, College of Veterinary Medicine, Kansas State

7 University, Manhattan, KS 66506-5701.

$8 \quad{ }^{\mathrm{b}}$ Department of Clinical Sciences, College of Veterinary Medicine, Kansas State University,

9 Manhattan, KS 66506-5701.

$10{ }^{\mathrm{c}}$ Department of Statistics, College of Arts and Sciences, Kansas State University, Manhattan, KS

$11 \quad 66506-5701$.

12 d Department of Geography, College of Arts and Sciences, Kansas State University, Manhattan, 13 KS 66506-5701.

14

$15{ }^{*}$ Corresponding author:

16 R.K. Raghavan

17 Kansas State Veterinary Diagnostic Laboratory

18 College of Veterinary Medicine

19 Manhattan, KS. 66506-5701

20 Tel.: 7855322492

$21 \quad$ Fax., 7855324481

22 E-mail address: rkraghavan@vet.k-state.edu 


\section{Abstract:}

Associations of housing, population, and agriculture census variables, and presence near

26 public places were retrospectively evaluated as potential risk factors for canine leptospirosis

27 using Geographic Information Systems (GIS). The sample population included 94 dogs positive

28 for leptospirosis based on a positive polymerase chain reaction test for leptospires on urine,

29 isolation of leptospires on urine culture, a single reciprocal serum titer of 12,800 or greater, or a

30 four-fold rise in reciprocal serum titers over a 2 to 4 week period; and 185 dogs negative for

31 leptospirosis based on a negative polymerase chain reaction test and reciprocal serum titers less

32 than 400. Multivariable logistic regressions revealed different risk factors among different census

33 units; however, houses lacking complete plumbing facilities $[\mathrm{OR}=2.80,95 \%$ C.I $=1.82,4.32$

34 (census unit, block group); $\mathrm{OR}=1.36,95 \%$ C.I. $=1.28,1.45$ (census tract); and, $\mathrm{OR}=3.02,95 \%$

35 C.I. $=2.60,3.52$ (county)]; and poverty status by age $(18-64)[\mathrm{OR}=2.04,95 \%$ C.I $=1.74,2.39$

36 (block group); $\mathrm{OR}=1.53,95 \%$ C.I. $=1.41,1.67$ (census tract); and, $\mathrm{OR}=1.62,95 \%$ C.I. $=1.50$,

371.76 (county)] were consistent risk factors for all census units. Living within $2500 \mathrm{~m}$ of a

38 university/college and parks/forests were also significantly associated with leptospirosis status in

39 dogs. Dogs that live under these circumstances are at higher risk for leptospirosis and pet owners 40 should consider vaccination.

42 Key words: Leptospirosis, Canine, Socio-economic status, Modifiable Areal Unit Problem 43 (MAUP), Geographic Information Systems (GIS). 
Leptospirosis is a worldwide zoonotic disease that can create disease in and be

49 transmitted by rodents, small mammals, dogs, swine, and cattle, among others, and has been

50 attributed to more than 200 pathogenic serovars from the genus Leptospira, although in any one

51 geographic area disease is typically limited to a few serovars (Greene et al., 2006). Three basic

52 epidemiological patterns of transmission are described for leptospirosis (Faine et al., 2000). The

53 first, transmission to humans (and presumably dogs) in temperate climates occurs through direct

54 contact with cattle and pigs. The second pattern is associated with tropical climates, but in

55 contrast to the first involves many serovars and large numbers of reservoir species infecting

56 humans and animals. The third pattern, which concerns urban environments and is of importance

57 to humans and dogs, is typically associated with rodent transmission of limited serovars,

58 although other peridomestic wildlife, such as raccoons and opossums may play a role (Feigin et

59 al., 1973; Demers et al., 1985; Vinetz et al., 1996; Richardson and Gauthier, 2003).

60 In studies that are mainly reported from South America the incidences of leptospirosis in

61 humans have been associated with socio-economic and demographic characteristics of a society

62 such as income, literacy, housing and population density (Veras et al., 1985; Everard et al., 1989;

63 Bakoss, 2007; Cruz et al., 2009). Martins Soares et al., (2010) explored several socio-economic

64 and demographic characteristics of Sao Paulo, Brazil with historical human leptospirosis cases

65 and found significant associations with average monthly income, literacy rate, and number of

66 people living in a household, among other factors. Likewise, education, income, housing type,

67 and number of people living per household were risk factors for human leptospirosis in a

68 different study from urban Recife in Brazil (Oliveira et al., 2009). Many of the measures of

69 socio-economic and housing conditions differ in the U.S. compared to Brazil and other South 
70 American countries and, to our knowledge no study has previously addressed the influence of pet

71 owner socio-economic and demographic characteristics with canine leptospirosis in the U.S.

72 A pet owner's education, age, and income, and population density and the housing

73 characteristics of a neighborhood in which dogs reside are some factors that may have an impact

74 on the health status due to the similarities in living conditions shared by pets and their owners.

75 Other factors that may influence canine leptospirosis incidence in urban settings include

76 proximity to public or open land that provide recreational opportunities (Ghneim et al., 2007)

77 and living within newly urbanized areas (Ward et al., 2004), and agriculture and livestock related

78 activities in the region (Ward et al., 2004).

Associations of socio-economic and demographic features to animal and human

80 infectious diseases can be quantitatively evaluated using spatial analysis and geoprocessing

81 capabilities of a Geographic Information System (GIS). In an earlier study, using GIS and

82 publicly available land cover datasets we found that urban areas in general and medium and high

83 density residential areas in particular are significant risk factors for leptospirosis when land

84 use/land cover area surrounding up to $2500 \mathrm{~m}$ from dogs' residences were analyzed (Raghavan et

85 al., 2011). However, variables representing specific socio-economic or demographic

86 characteristics of urban land use were not included in that study nor have they been analyzed in

87 other published literature.

The objectives of this retrospective case-control study were to investigate which urban

89 characteristics, specifically socio-economic and human demographic factors could be potential

90 risk factors for canine leptospirosis in Kansas and Nebraska. Further, living within the proximity

91 of certain public areas was also evaluated as potential risk factors for leptospirosis. 
94 2.1. Case selection:

95 The medical records of all dogs from Kansas and Nebraska that had urine polymerase

96 chain reaction (PCR) testing for leptospirosis performed at the Kansas State Veterinary

97 Diagnostic Laboratory (KSVDL) between February, 2002 and December, 2009 were

98 retrospectively reviewed. When available, additional test results were included, specifically the

99 results of leptospiral serology and urine culture for leptospirosis. A positive case was defined by

100 a positive urine PCR or a negative urine PCR and any one of the following: isolation of

101 leptospires on urine culture, a single reciprocal serum titer $\geq 12,800$, or a four-fold rise in the

102 reciprocal convalescent serum titer. Dogs were deemed negative controls if the urine PCR was

103 negative and reciprocal serum titers were $<400$.

104

105 2.2. Molecular diagnostic testing:

106 Urine samples for PCR were handled for DNA isolation as previously reported (Harkin et

107 al., 2003). DNA samples were subjected to the semi-nested, pathogenic Leptospira PCR assay

108 described by Woo et. al., (1997) that amplifies a conserved region of the 23S rDNA, with minor

109 modifications. A unique Taqman probe was incorporated to distinguish pathogenic Leptospira

110 from saprophytic serovars. This test has been commercially available through the KSVDL since 1112002.

112

113 2.3. Serological testing:

114 The microscopic agglutination test was performed on all blood samples submitted to the

115 KSVDL for leptospiral serological testing. The test was performed for serovars Canicola, 
116 Bratislava, Pomona, Icterohemorrhagiae, Hardjo, and Grippotyphosa.

118 2.4. Leptospiral culture:

119 Urine culture was performed by inoculating $1 \mathrm{ml}$ of urine obtained by cystocentesis 120 immediately into $10 \mathrm{ml}$ of liquid Ellinghausen-McCullough (EM) media, gently vortexing this

121 inoculation and transferring $1 \mathrm{ml}$ of this into another $10 \mathrm{ml}$ of liquid EM media. One milliliter of 122 each dilution (1:10 and 1:100) was then subsequently inoculated into separate $10 \mathrm{ml}$ of semi123 solid EM media. All tubes were incubated at $30^{\circ} \mathrm{C}$ in an ambient atmosphere incubator and 124 evaluated for evidence of growth weekly.

2.5. Demographic information:

Medical records were reviewed in order to obtain the following information: the patient's 128 age, rounded up to the nearest month, at the time of sample submission; the date of sample 129 submission; the client's street address at the time of sample submission, breed and sex.

131 2.6. Geocoding:

132 Household addresses with information pertaining to house number, street, city, state and 133 zip code were provided by clients at the time specimens for leptospirosis testing were submitted. 134 Addresses were retrospectively verified for their accuracy either by using MapQuest (Map Quest. 135 America Online, Denver, CO) or Google Maps (Google Inc., Mountain View, CA) and/or calling 136 telephone numbers provided by clients. Geographic coordinates for these addresses were derived 137 using a geocoding tool in ArcMap 9.3.1 software and US Census 2007 TIGER (Topographically 138 Integrated Geographic Encoding and Referencing system) shapefile with street level address 
139 information (US Census Bureau, 2011). The geographic coordinates for unmatched addresses

$140(8 \%)$ were obtained using Google Earth software (version No: 5.2.1.1329) (Google Inc.,

141 Mountain View, CA). In all, geographic coordinates for 94 (out of 97) cases and 185 (out of 197)

142 control data points in Kansas and Nebraska were obtained.

144 2.7. Host factors

145 Observations were grouped into five age groups $<1 \mathrm{y}, 1$ to $4 \mathrm{y}, 4$ to $7 \mathrm{y}, 7$ to $10 \mathrm{y}$ and $>$ $14610 \mathrm{y}$; two sexes and 77 individual breeds, including mixed breeds and unknown or unspecified 147 breeds were kept without grouping as a categorical variable.

149 2.8. Projection and data storage:

150 GIS datasets used in this study were projected (or re-projected from their original spatial 151 reference) in to the USA Contiguous Equal Area and Equidistant Conic Projections, both of 152 which were based on the Geographic Coordinate System North American 1983 Geographic 153 Datum. All original, intermediate and processed GIS data were stored in a SQL Server/ESRI 154 ArcSDE 9.3.1 Geodatabase.

156 2.9. Census data:

U.S. Census 2000 data on population and housing were obtained in the form of

158 Summary File 3 (SF-3) tables from the U.S. Census Bureau (U.S. Census Bureau, 2011).

159 Identical census attribute information for Kansas and Nebraska were gathered at three

160 geographic levels or census units at which census data were aggregated by the US Census

161 Bureau: block groups (containing between 600 and 3,000 people within a county), census tracts 
162 (containing between 1,500 and 8,000 people intended to represent neighborhoods), and counties.

163 GIS data files for block groups, tracts and counties were obtained from the ESRI Street

164 Map data based on US Census Bureau 2000 census information. From the Summary File -3 (SF-

165 3) tables, 33 housing and 37 population related variables (Table 1) were extracted for each

166 census unit by spatial query and joined to the census shapefiles using the common Federal

167 Information Processing Standards (FIPS) codes. Each census category included several

168 independent variables and they were evaluated separately in the study. The geocoded addresses

169 of cases/controls were overlaid in ArcMap with block group, census tract, and county shapefiles

170 in three separate operations, and the number of cases/controls that were within census units were

171 recorded separately using a spatial join procedure in ArcMap.

172

173 2.10. Agricultural census:

174 Agricultural census data for Kansas and Nebraska was obtained per county from the

175 USDA National Agricultural Statistics Service (NASS) (USDA, 2011). Six county level

176 agricultural census data were obtained from NASS in a tabular format, including the total

177 number of cattle farms, total number of swine farms, the total number of dairy cattle, total

178 number of beef cattle, the number of pigs and the number of hogs per county in year 2007.

180 2.11. Presence near public places:

181 Polygon areas representing ten different public places around cities, including golf

182 courses, hospitals, industrial parks, primary/secondary schools, shopping centers, sports

183 stadiums, and local, county, and state parks/forests, and universities/colleges within $5000 \mathrm{~m}$ from

184 dogs' homes in the study region were obtained from the US Census 2000 TIGER/Line dataset. 
185 Buffered areas extending $2500 \mathrm{~m}$ from the boundaries of public places were created and 186 cases/controls located completely outside (coded ' 0 ') and within (coded ' 1 ') the buffers were 187 recorded independently for each public place type. Ten variables, representing location within $188 \quad 2500 \mathrm{~m}$ from every public place were thus derived.

2.12. Data organization and statistical analysis:

All census data were originally stored in a Microsoft Access 2010 (Microsoft, Redmond,

192 CA) database and later as ESRI shapefiles during spatial analysis. The number of cases/controls

193 within and outside newly urbanized areas, and the distances to public places from cases/control

194 locations were stored as ESRI shapefiles. All numerical data were stored in Microsoft Excel

1952010 (Microsoft, Redmond, CA) prior to statistical analyses conducted using SAS software (SAS

196 Institute, Cary, NC) or R Statistical Package 2.11.1 (R Core Development Team, 2011) when

197 specified. During the exploratory spatial analysis of case/control locations in the study region

198 clustering among cases and controls were evaluated using Cuzick-Edwards $K^{\text {th }}$ neighbor statistic

199 (Cuzick and Edwards, 1990) within six major cities in the study region, including Manhattan,

200 Wichita, Topeka and Kansas City in Kansas, and Omaha and Lincoln in Nebraska. Four

201 neighbors were included in the analysis for cluster detection.

202 Odds ratios and $95 \%$ confidence intervals derived using logistic regressions were used to 203 determine associations of canine leptospirosis status with independent variables. There were a 204 total of 33 housing related variables and 37 population related variables at block group, census 205 tract, and county levels; 6 agricultural census variables at county level, and 10 variables 206 representing proximity to different public places. Variable screening among all variables was 207 done by fitting univariable logistic models and those variables with a $P$-value $\leq 0.1$ were selected 
208 for further analysis; however, care was taken not to remove variables that were deemed clinically

209 relevant (Hosmer and Lemeshow, 2000; Ward et al., 2004; Raghavan et al., 2011).

210 Multicollinearity was tested among screened variables by estimating the variable inflation factor

211 (VIF) using the proc reg/tol vif option in SAS (SAS Institute Inc., Cary, NC). All variables with a

212 VIF value of 10 or above were considered to indicate multicollinearity (Allison, 1999).

213 Multivariable logistic regression was conducted using screened variables in three separate

214 steps with variables from each census unit at a time along with variables from other groups

215 (agricultural census variables at county level and, location within $2500 \mathrm{~m}$ from public places).

216 Observations for all census variables were kept in their original measurement units and were

217 continuous. Observations for presence within $2500 \mathrm{~m}$ from public places were in categorical

218 format scored as ' 0 ' if absent and ' 1 ' if present. Interaction terms were not included in the 219 models.

220 Multivariable logistic models with events/trials operand were fit using the stepwise

221 selection procedure in which a significance level, $P \leq 0.05$ was used for a variable to be retained

222 and $P \geq 0.1$ to be removed from the model (SAS, 2011). Logistic models were ranked using

223 Akaike Information Criterion (AIC) and the model with the lowest AIC value was deemed to be

224 the best fitting model. Any confounding effect of host factors, age $(<1 \mathrm{y}$ old as reference level),

225 sex (female as reference level), and breed (unknown or unspecified as reference level) was

226 estimated by adding them one at a time to the final logistic model, and a $10 \%$ or more change in

227 coefficient values of independent variables were considered to indicate confounding due to that

228 particular factor, in which case adjusted odds ratios and their 95\% confidence intervals were

229 recorded. Linearity assumption for logit in final models was assessed using Box-Tidwell test

230 (Box and Tidwell, 1962). Model adequacy was tested using chi-squared goodness-of-fit test $(P<$ 
2310.05 indicated poor fit), and predictive ability measured by deriving the Area under Receiver's 232 operator's characteristic (ROC) curve value.

233 Spatial autocorrelation if present in the case/control data could lead to the violation of

234 underlying logistic regression assumptions (that the samples are independent and identically

235 distributed) and will yield incorrect parameter estimates and error term. If the parameters in the

236 multivariable model did not account for autocorrelation then the residuals of the model will

237 reveal autocorrelation and need to be verified (Robinson, 2000). A monte-carlo test based on the

238 empirical variogram of residuals and their spatial envelopes (generated by permutations of data

239 values across spatial locations) was used to check for spatial autocorrelation using the geoR

240 library of R Statistical Package 2.11.1 (Ribeiro and Diggle, 2001; Ribeiro et al., 2003).

242 3. Results:

243 There were 94 dogs that were identified as cases based on a positive PCR ( $n=90$ dogs),

244 isolation of leptospires from the urine $(n=1)$, a single reciprocal titer $\geq 12,800(n=2)$, or a four-

245 fold rise in serum reciprocal titers $(n=1)$. Of the dogs that were PCR positive, serology was not

246 performed in 22 dogs, 7 dogs had a negative acute titer with no convalescent titer performed, and

24761 dogs had concurrent elevated titers to one or more serovar. There were 185 control dogs that

248 had a negative PCR and a reciprocal serum titer of $<400$.

249 Among 94 cases and 185 controls evaluated in this study, a majority had their physical

250 addresses located in the city of Wichita [33.68\%, 28.81\% (case, control)] followed by Manhattan

$251(13.82 \%, 19.45 \%)$, Lincoln $(10.52 \%, 8.96 \%)$, Omaha (9.47\%, 5.24\%), Kansas City (6.31\%,

$2524.62 \%)$ and Topeka (6.31\%, 5.94\%). All remaining cases (19.89\%) and controls (26.98\%) had

253 rural addresses or they were from smaller cities in the study region. 
Since there could be a bias in case reporting to hospitals from certain neighborhoods than

255 others due to income differences, it was essential to verify if cases/controls showed any tendency

256 to cluster in any of the major cities in the study region. However, no clustering was observed in

257 any of the cities (where income levels among neighborhoods could vary). The Cuzick-Edwards

258 estimates for case locations in Manhattan $(P=0.19)$, Wichita $(P=0.41)$, Topeka $(P=0.24)$,

259 Kansas City $(P=0.28)$, Lincoln $(P=0.31)$, and Omaha $(P=0.47)$ did not indicate any

260 clustering. Similarly, the Cuzick-Edwards estimates for control locations in Manhattan $(P=$

261 0.05), Wichita $(P=0.26)$, Topeka $(P=0.36)$, Kansas City $(P=0.19)$, Lincoln $(P=0.18)$, and

262 Omaha $(P=0.22)$ did not indicate any clustering as well.

263 There were differences in the number and types of significant housing and population

264 variables identified in logistic models fit with covariates from different census units (Tables 2-

265 4). When block group level housing and population variables were analyzed along with

266 agricultural census and public places variables, the housing related variables significantly

267 associated with leptospirosis status in the logistic model were; the total number of structures built

268 during the years (1940-1949) and the number of households lacking complete plumbing

269 facilities (houses lacking hot and cold piped water, a flush toilet, and a bathtub or shower).

270 Significant population related covariates associated with leptospirosis status in the logistic model

271 were poverty status in 1999 by age (18-64) (number of individuals in the age group 18-64 that

272 were below poverty line the year 1999). Presence within $2500 \mathrm{~m}$ from university/college

273 campuses and park/forest areas were significantly associated with leptospirosis status in dogs

274 (Table 2).

275 When census tract level housing and population variables were analyzed along with 276 agricultural census and public places variables, the only housing related covariate significantly 
277 associated with leptospirosis status in the logistic model was the number of households lacking 278 complete plumbing facilities, and the only population related covariate significantly associated

279 with leptospirosis status in the logistic model was poverty status in 1999 by age (18-64).

280 Presence within $2500 \mathrm{~m}$ from university/college campuses and park/forest areas were

281 significantly associated with leptospirosis status in dogs (Table 3).

282 Using county level housing and population variables along with agricultural census and 283 public places variables, the housing related covariates significantly associated with leptospirosis 284 status in the logistic model were the number of households lacking complete plumbing facilities 285 and the number of owner occupied homes. The only population related covariate significantly 286 associated with leptospirosis status in the logistic model was poverty status in 1999 by age (18287 64) (Table 4). Presence within $2500 \mathrm{~m}$ from university/college campuses was marginally 288 significant, and park/forest areas were significantly associated with leptospirosis status in dogs. 289 Two agricultural census variables (the density of cattle farms, and the number of beef 290 cattle per county) were significantly $(P<0.1)$ associated with leptospirosis status but were not 291 significant in the multivariable logistic model. For all models described above, no other 292 covariates were found to be significant and/or found to improve the model fit when added. The 293 chi-square deviance goodness of fit test did not indicate any model inadequacy, and non-linearity 294 in logit and residual autocorrelation was absent. Confounding effects of age, breed, and sex were 295 not noted for any models.

296 4. Discussion:

297 The lack of clustering of cases and controls in any of the major cities in the study region 298 indicate a lack of sample bias for low income vs. high income neighborhoods in the study 299 population. In addition, the issue of referral bias is usually encountered in case-control studies 
with the diagnostic laboratories receiving relatively higher numbers of cases from immediate

301 neighboring areas due to proximity and familiarity with the facility. However, the referrals in this

302 study originated from all major cities and rural areas in the study region, and in addition, $25.6 \%$

303 of the study population included dogs that were diagnosed by the primary care veterinarian

304 outside KSVDL. The number of days that the dogs lived in their owner's household was not

305 provided to us during case submissions. For the purposes of this study, it was assumed that the

306 dogs spent most of their lives in their owners' households except for those times spent outside

307 during recreation and/or supervised exercise.

308 Demographic and socio-economic data collected by the U.S. Census Bureau and other

309 agencies are highly relevant to public health and epidemiological research. However, such data

310 are most commonly aggregated at the level of administrative boundaries or census/areal units

311 (Fig. 1). It has been well documented that the choice of areal unit could affect the strength and

312 significance of statistical associations and renders the results difficult to compare with other

313 studies. This is known as the Modifiable Areal Unit Problem (MAUP) (Openshaw, 1984; Unwin,

314 1996). Currently there are no solutions to fully overcome the effects of MAUP and related

315 methodological issues have not yet been adequately addressed. Recommendations have been

316 made to minimize MAUP effects in statistical inference by analyzing the aggregated covariates

317 in hierarchical levels of areal units from the finest spatial resolution possible to a coarser

318 resolution and to verify consistent model results (Fotheringham, 1989; Ratcliffe and McCullagh,

319 1999; Diez Roux, 2000). Three hierarchical levels of census units commonly used in

320 epidemiological studies were used in this study for identical housing and population covariates.

321 There were differences in the significant census variables in multivariable logistic models

322 at different areal levels (block group, census tract, and county) likely due to MAUP; however, 
323 the number of households that lack plumbing facilities and the number of individuals in the 18

32464 year age group that are below poverty line were consistent risk factors in all areal units. These 325 and other housing and population related variables associated with canine leptospirosis status at 326 independent areal units are indicative of lower pet-owner socio-economic conditions and lower 327 housing standards, which are likely related. The findings reported here are similar to some of the 328 risk factors reported in studies from Brazil (Oliveira et al., 2009; Barcellos et al., 2000; Veras et

329 al., 1985) where more canine and human leptospirosis cases were shown to originate from poorer 330 neighborhoods. As in this study, the vaccination status of dogs included in the studies originating

331 from Brazil are not clear but dogs could be at higher risk in such urban environments due to pet

332 owners failing to vaccinate their dogs and/or higher prevalence of leptospirosis in the

333 environment due to substandard housing and other neighborhood conditions.

334 Among all public lands within an area covering $5 \mathrm{~km}$ from 2000 census city boundaries,

335 proximity to colleges/university campuses and state parks/forests were significantly associated

336 with leptospirosis status (when analyzed along with county level census data, the significance

337 value of college/university campus was slightly over $\alpha=0.05$ ). Land use areas representing

338 parks/forests and college/universities are similar in that they provide ample open spaces for

339 canine recreation and are places where high dog-to-dog and wild mammal contact could occur.

340 However, parks/forests are relatively well drained areas compared to college/universities that

341 have built up areas such as parking-lots and pavements and there is potential for water run-off,

342 flooding and overflow from streams nearby. Therefore, the risk of public places such as

343 college/universities and similar environments may be due to flooding events.

$344 \quad$ An outbreak in human leptospirosis in a university campus was reported after flooding

345 and embankment overflow within the campus (Gaynor et al., 2007), and one human case of 
346 leptospirosis was diagnosed after a similar flood event on another university campus (Park et al.,

347 2006). Precipitation and flooding have been associated with increased leptospirosis incidence

348 (Kawaguchi et al., 2008; Ward et al., 2004; Liverpool et al., 2008) and flood-prone or frequently

349 flooded areas are risk factors for human and canine leptospirosis (Morshed et al., 1994; Karande

350 et al., 2002; Batista et al., 2005). In addition, college/university campuses in the study region are

351 generally found in high density neighborhoods where housing is relatively older and the resident

352 population comprise higher number of students that likely change year to year and whose income

353 levels are typically low, factors which could play a role in higher transmission rates.

354 Proximity to open sewer and public waste disposal sites has been associated with human

355 leptospirosis from other countries (Oliveira et al., 2009; Krojgaard et al., 2009; Sarkar et al.,

356 2002). In the U.S., open sewer systems are not permitted by legislation unless they are within

357 treatment plants. Public waste disposal sites and landfills in the study region were located

358 beyond $5000 \mathrm{~m}$ from any case/control location and away from the city boundaries; therefore,

359 geographic features representing such areas were not included in the analysis. Proximity to storm

360 water drainage systems in the study region, some of which are open to the environment was not

361 associated with leptospirosis status. It is possible that the open storm water drainage systems in

362 the study region are free of leptospira, inaccessible for direct contact, or the peridomestic animal

363 movement around these areas could be minimal.

364 5. Conclusion:

365 Poverty status among people in 18-64 year age group, houses that lack plumbing

366 facilities, and proximity to public parks, college/universities, and newly urbanized areas are risk

367 factors for canine leptospirosis in Kansas and Nebraska, and likely other regions in the world as

368 well. Pet owners living under such neighborhood characteristics and treating veterinarians should 
369 consider vaccination for their dogs in order to prevent leptospirosis.

370

371

372

373

374

375

376

377

378

379

380

381

382

383

384

385

386

387

388

389

390

391 
392 Acknowledgements:

393 Support for this research was partly provided by Kansas State University Diagnostic

394 Laboratory and NSF (grant No. 0919466, Collaborative Research: EPSCoR RII Track 2

395 Okhlahoma and Kansas: A cyber Commons for Ecological Forecasting). The authors thank Dr.

396 Michael Ward, Faculty of Veterinary Science, University of Sydney, Dr. John Keller, Department

397 of Regional and Community Planning, Kansas State University, Dr. Keith Gido, Division of

398 Biology, Kansas State University, and two anonymous reviewers for their valuable suggestions to

399 improve earlier versions of the manuscript.

400

401

402

403

404

405

406

407

408

409

410

411

412

413

414 
416 Allison, P.D., 1999. Logistic regression using the SAS system. Theory and application. SAS 417 Institute Inc., Cary, NC.

418 Bakoss, P., Machacova, E., Jarekova, J., 2007. Results of surveillance of human leptospirosis. 419 Epidemiologie Mikrobiologie Imunologie 56, 140-149.

420 Barcellos, C., Sabroza, P.C., 2000. Socio-environmental determinants of the leptospirosis 421 outbreak of 1996 in western Rio de Janeiro: a geographical approach. International 422 Journal of Environmental Health Research 10, 301-313.

423 Batista, C.S.A., Alves, C.J., Azevedo, S.S., Vasconcellos, S.A., Morais, Z.M., Clementino, I.J., Alves, F.A.L., Lima, F.S., Neto, J.O.A., 2005. Seroprevalence and risk factors for leptospirosis in dogs from Campina Grande, State of Paraiba, Brazil. Arquivo Brasileiro de Medicina Veterinaria e Zootecnia 57, 179-185.

Box, G.E.P., Tidwell, P.W., 1962. Transformation of the independent variables. Technometrics 428 $4,531-550$.

Cruz, L.S., Vargas, R., Lopes, A.A., 2009. Leptospirosis: a worldwide resurgent zoonosis and important cause of acute renal failure and death in developing nations. Ethnicity \& Statistical Society Series B 52, 73-104. 
434 Demers, R.Y., Frank, R., Demers, P., Clay, M., 1985. Leptospiral exposure in Detroit rodent control workers. American Journal of Public Health 75, 1090-1091.

436
Diez Roux, A. V., 2000. Multilevel analysis in public health research. Annual Review of Public Health 21, 171-192.

Everard, C.O.R., Hayes, R.J., Edwards, C.N., 1989. Leptospiral infection in school-children from Trinidad and Barbados. Epidemiology and Infection 103, 143-156.

Faine, S., Adler, B., Bolin, C., Perolat, P., 2000. Leptospira and leptospirosis. $2^{\text {nd }}$ edition. MediSci, Melbourne.

Feigin, R.D., Lober, L.A.J., Anderson, D., Pickering, L., 1973. Human leptospirosis from immunized dogs. Annals of Internal Medicine 79, 777-785.

Fotheringham, S., 1989. Scale independent spatial analysis. In: Goodchild, M.F., Gopal, S., (Eds.), The accuracy of spatial databases. 1994Taylor and Francis, London, pp. 221-228.

Gaynor, K., Katz, A.R., Park, S.Y., Nakata, M., Clark, T.A., Effler, P.V., 2007. Leptospirosis on Oahu: An outbreak associated with flooding of a University Campus. American Journal of Tropical Medicine and Hygiene 76, 882-885.

Ghneim, G.S., Viers, J.H., Chomel, B.B., Kass, P.H., Descollonges, D.A., Johnson, M.L., 2007. Use of a case-control study and geographic information systems to determine environmental and demographic risk factors for canine leptospirosis. Veterinary Research 38, 37-50. 
453 Greene, C.E., 2006. Laboratory diagnosis of canine leptospirosis and babesiosis. 24th Annual ACVIM Forum, Louisville, Kentucky, USA, 31 May-3 June, 2006., 490-491.

455

Harkin, K.R., Roshto, Y.M., Sullivan, J.T., 2003. Clinical application of a polymerase chain reaction assay for diagnosis of leptospirosis in dogs. Journal of the American Veterinary Medical Association 222, 1224-1229.

Hosmer, D.W., Lemeshow, S., 2000. Model-building strategies and methods for logistic regression. In: Applied Logistic Regression. Second Edition. Eds: Hosmer, D.W., Lemeshow, S. pp: 91-142. John Wiley \& Sons, New York.

Karande, S., Kulkarni, H., Kulkarni, M., De, A., Varaiya, A., 2002. Leptospirosis in children in Mumbai slums. Indian Journal of Pediatrics 69, 855-858.

Kawaguchi, L., Sengkeopraseuth, B., Tsuyuoka, R., Koizumi, N., Akashi, H., Vongphrachanh, P., Watanabe, H., Aoyama, A., 2008. Seroprevalence of leptospirosis and risk factor analysis in flood-prone rural areas in Lao PDR. American Journal of Tropical Medicine and Hygiene 78, 957-961.

Krojgaard, L.H., Villumsen, S., Markussen, M.D.K., Jensen, J.S., Leirs, H., Heiberg, A.C., 2009. High prevalence of Leptospira spp. in sewer rats (Rattus norvegicus). Epidemiology and Infection 137, 1586-1592.

Liverpool, J., Francis, S., Liverpool, C.E., Dean, G.T., Mendez, D.D., 2008. Leptospirosis: case reports of an outbreak in Guyana. Annals of Tropical Medicine \& Parasitology 102, 239245. 
473 Martins Soares, T.S., Dias de Oliveira Latorre, M.d.R., Laporta, G.Z., Buzzar, M.R., 2010.

474 Spatial and seasonal analysis on leptospirosis in the municipality of Sao Paulo,

475 Southeastern Brazil, 1998 to 2006. Revista de Saude Publica 44, 283-291.

476 Morshed, M.G., Konishi, H., Terada, Y., Arimitsu, Y., Nakazawa, T., 1994. Seroprevalence fo

477 leptospirosis in rural flood prone district of Bangladesh. Epidemiology and Infection 112,

$478 \quad 527-531$.

479 Oliveira, D.S.C., Guimaraes, M.J.B., Portugal, J.L., Medeiros, Z., 2009. The socio-demographic,

480 environmental and reservoir factors associated with leptospirosis in an urban area of

481 north-eastern Brazil. Annals of Tropical Medicine and Parasitology 103, 149-157.

482 Openshaw, S., 1984. The modifiable areal unit problem, Concepts and Techniques in Modern

483 Geography No. 28, Geo Books, Norwich.

484 Park, S.Y., Effler, P.V., Nakata, M., Sasaki, D., Katz, A.R., Clark, T.A., Gaynor, K., 2006.

485 Leptospirosis after flooding of a university campus - Hawaii, 2004. Morbidity and

$486 \quad$ Mortality Weekly Report 55, 125-127.

487 R Core Development Team, 2011. R: A language and environment for statistical computing,

488 reference index version 2.11.1. R Foundation for Statistical Computing, Vienna, Austria.

489 Raghavan, R., Brenner, K., Higgins, J., Van der Merwe, D., Harkin, K.R., 2011. Evaluations of

490 land cover risk factors for canine leptospirosis: 94 cases (2002-2009). Preventive

$491 \quad$ Veterinary Medicine 101, 241-249.

492 Ratcliffe, J. H., McCullagh, M. J., 1999. Hotbeds of crime and the search for spatial accuracy 
494 Ribeiro, P.J., Christensen, O.F., Diggle, P.J., 2003. geoR and geoRglm: Software for model 495 based geostatistics. In: Hornik, K., Leisch, F., Zeileis, A. (Eds.), 3rd International Workshop on Distributed Statistical Computing (DSC 2003), Vienna, Austria.

Ribeiro, P.J., Diggle, P.J., 2001. geoR: a package for geostatistical analysis., R-News. Vienna, pp. $15-18$.

Richardson, D.J., Gauthier, J.L., 2003. A serosurvey of leptospirosis in Connecticut peridomestic wildlife. Vector-Borne and Zoonotic Diseases 3, 187-193.

Robinson, T.P., 2000. Spatial statistics and geographical information systems in epidemiology and public health. Advances in Parasitology 47, 81-128.

SAS, 2011. SAS/STAT(R) 9.2 User's Guide. Second Edition. http://support.sas.com/documentation/cdl/en/statug/63033/HTML/default/viewer.htm\#sta

Sarkar, U., Nascimento, S.F., Barbosa, R., Martins, R., Nuevo, H., Kalafanos, I., Grunstein, I., Flannery, B., Dias, J., Riley, L.W., Reis, M.G., Ko, A.I., 2002. Population-based casecontrol investigation of risk factors for leptospirosis during an urban epidemic. American Journal of Tropical Medicine and Hygiene 66, 605-610. 
513 Unwin, D. J., 1996. GIS, spatial analysis and spatial statistics, Progress in Human Geography 20, $514 \quad 540-441$.

515 Veras, F.M.F., Rouquayrol, M.Z., Gomes, I.L.D.P., 1985. Epidemiological study of the 516 leptospirosis cases observed in Fortaleza, Brazil during the epidemic of . Revista de 517 Medicina da Universidade Federal do Ceara 25, 55-62.

518 Vinetz, J.M., Glass, G.E., Flexner, C.E., Mueller, P., Kaslow, D.C., 1996. Sporadic urban 519 leptospirosis. Annals of Internal Medicine 125, 794-798.

520 Ward, M.P., Guptill, L.F., Wu, C.C., 2004. Evaluation of environmental risk factors for 521 leptospirosis in dogs: 36 cases (1997-2002). Journal of the American Veterinary Medical Association 225, 72-77.

523 Woo, T.H.S., Patel, B.K.C., Smythe, L.D., Symonds, M.L., Norris, M.A., Dohnt, M.F., 1997.

524 Identification of pathogenic Leptospira genospecies by continuous monitoring of 525 fluorogenic hybridization probes during rapid-cycle PCR. Journal of Clinical $526 \quad$ Microbiology 35, 3140-3146. 
531 Table 1. Population and housing variables from US Census Bureau SF-3 data evaluated in the

532 study.

533 Census category Independent variables*

$534 \quad$ Housing

$535 \quad$ Housing Units

Total housing units.

$536 \quad$ Urban and rural

Urban, rural, farm, nonfarm.

$537 \quad$ Tenure

Owner occupied, renter occupied.

$538 \quad$ Race of householder

White alone, Black or African American alone, American

539

Indian and Alaska Native alone, Asian alone, Native

540

Hawaiian and Other Pacific Islander alone, some other race

541

alone, two or more races.

$542 \quad$ Household size

1-person, 2-person, 3-person, 4-person, 5-person, 6-

person, 7-or-more person household.

544 Median number of rooms

Median number of rooms.

$545 \quad$ Year structure built

Built 1999 to March 2000, 1995 to 1998,1990 to 1994 , 546 1980 to 1989,1970 to 1979,1960 to 1969,1950 to 1949 , 1940 to 1949 , Built 1939 or earlier.

$548 \quad$ Plumbing facilities

Complete plumbing facilities, lacking complete plumbing 549 facilities. 
552 Population

553 Family size

$554 \quad$ Urban and rural

$555 \quad$ Race

556

557

558

559 Household income in 1999

560

561

562

563

564

565

566

567

568

569

570

571

572
Total population.

Average family size

Urban, rural, farm, nonfarm.

White alone, Black or African American alone, American

Indian and Alaska Native alone, Asian alone, Native

Hawaiian and Other Pacific Islander alone, some other race alone, two or more races.

Less than $\$ 10,000, \$ 10,000$ to $\$ 14,999$, and thirteen other variables representing $\$ 49,999$ incremental income thereof up to $\$ 199,999$, and $\$ 200,000$ or more.

Under 5 years, 5 years, 6 to 11 years, 12 to 17 years, 18 to 64 years, 65 to 74 years, 75 years and over.

* Observations for all the independent variables are counts, in continuous form, and recorded per areal unit (block group, tract or county). Each census category included several independent variables and they were evaluated separately in the study (for example, seven independent variables for the census category, Poverty status in 1999 by Age were evaluated).

Definitions of different census variables can be found from their source (U.S. Census Bureau) website at: http://www.census.gov/main/www/glossary.html 
573 Table 2. Results of multivariable logistic models $(P<0.05)$ with block group level housing and

574 population variables along with variables of agricultural census and public places associated with

575 canine leptospirosis status in the study region $(n=94$ cases, 185 controls).

576

577 Covariates

Estimate S.E OR 95\% C.I P-value

$578 \quad$ Year structures built (1940-1949)

$\begin{array}{lllll}0.80 & 0.20 & 2.22 & 1.50,3.30 & 0.00^{*}\end{array}$

579 Lacking complete plumbing facilities

$1.03 \quad 0.22 \quad 2.80 \quad 1.82,4.32 \quad 0.00^{*}$

580 Household income (30,000-34,999)

$\begin{array}{lllll}0.12 & 0.08 & 1.13 & 0.95,1.34 & 0.07\end{array}$

$581 \quad 6-$ person household

$\begin{array}{lllll}0.11 & 0.44 & 1.11 & 0.47,2.64 & 0.09\end{array}$

582 Poverty status in 1999 by age (18-64)

$\begin{array}{lllll}0.71 & 0.08 & 2.04 & 1.74,2.39 & 0.00^{*}\end{array}$

583 University/college

$\begin{array}{lllll}0.39 & 0.17 & 1.49 & 1.05,2.11 & 0.04^{*}\end{array}$

584 Park/forest

0.86

$\begin{array}{ll}0.36 & 2.37\end{array}$

$1.17,4.82 \quad 0.02^{*}$

585 C.I. - Confidence interval (low, high).

$586 *$ Significantly $(P<0.05)$ associated with leptospirosis status.

587 Area under ROC curve value $=0.71$.

588

589

590

591

592 Table 3. Results of multivariable logistic models $(P<0.05)$ with census tract level housing and 
593 population variables along with variables of agricultural census and public places associated with

594 canine leptospirosis status in the study region ( $\mathrm{n}=94$ cases, 185 controls).

595

596

Covariates

Estimate S.E OR 95\% C.I. P-value

597 6-person household

$\begin{array}{lllll}0.18 & 0.14 & 1.20 & 0.90,1.59 & 0.06\end{array}$

598 Lacking complete plumbing facilities

$\begin{array}{lllll}0.31 & 0.03 & 1.36 & 1.28,1.45 & 0.04^{*}\end{array}$

599 Poverty status in 1999 by age (18-64)

$\begin{array}{lllll}0.43 & 0.04 & 1.53 & 1.41,1.67 & 0.02^{*}\end{array}$

600 Poverty status in 1999 by age (65-74)

0.21

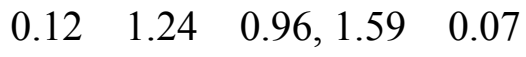

601 University/college

0.46

$0.18 \quad 1.581 .11,2.260 .03^{*}$

602 Park/forest

0.76

0.36

$2.151 .06,4.36 \quad 0.02^{*}$

603 C.I. - Confidence interval (low, high).

$604{ }^{*}$ Significantly $(P<0.05)$ associated with leptospirosis status.

605 Area under ROC curve value $=0.71$.

606

607

608

609

610

611 Table. 4. Results of multivariable logistic models $(P<0.05)$ with county level housing and

612 population variables along with variables of agricultural census and public places associated with 
613 canine leptospirosis status in the study region ( $\mathrm{n}=94$ cases, 185 controls).

614

615 Covariates $\quad$ Estimate $\quad$ S.E $\quad$ OR $95 \%$ C.I. $P$-value

616 Lacking complete plumbing facilities

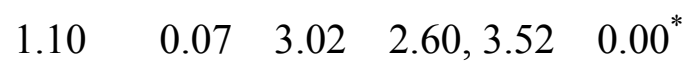

617 Owner occupied

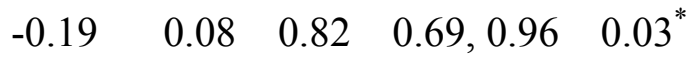

618 Poverty status in 1999 by age (18-64)

$\begin{array}{lllll}0.48 & 0.04 & 1.62 & 1.50,1.76 & 0.02^{*}\end{array}$

619 Household income (30,000-34,999)

$\begin{array}{lllll}0.97 & 0.66 & 2.64 & 0.72,9.67 & 0.07\end{array}$

620 University/college

$\begin{array}{lllll}0.35 & 0.18 & 1.42 & 0.99,2.03 & 0.05\end{array}$

621 Park/forest

0.82

$0.36 \quad 2.27 \quad 1.12,4.610 .03^{*}$

622 C.I. - Confidence interval (low, high).

$623{ }^{*}$ Significantly $(P<0.05)$ associated with leptospirosis status.

624 Area under ROC curve value $=0.67$.

625

626

627

628

629

630 
631

632 Distribution of case/control locations in counties of Kansas and Nebraska. Case locations were

633 distributed in 25 counties and control locations in 43 counties in the study region. Of the block

634 groups and census tracts (not shown in the map), cases and controls were distributed within 129

635 and 149 block groups respectively; and, within 90 and 103 census tract units respectively.

636

637

638

639

640

641

642

643

644

645

646

647

648

649

650

651

652

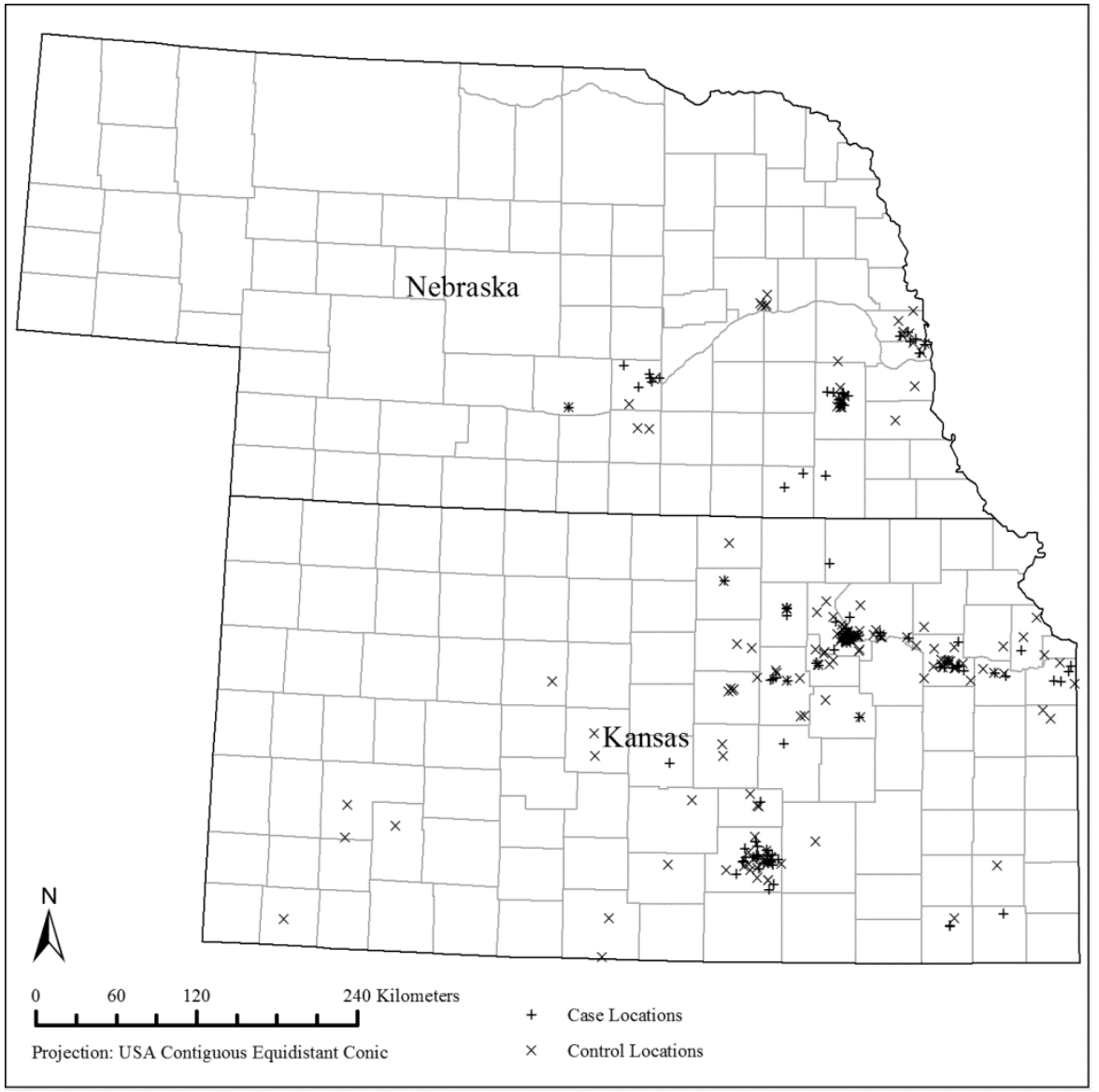

